\title{
A functional perspective on argumentation schemes
}

\author{
Adam Wyner \\ Department of Computing Science, University of Aberdeen, Aberdeen, UK \\ E-mail:azwyner@abdn.ac.uk
}

\begin{abstract}
In multi-agent systems (MAS), abstract argumentation and argumentation schemes are increasingly important. To be useful for MAS, argumentation schemes require a computational approach so that agents can use the components of a scheme to construct and present arguments and counterarguments. This paper proposes a syntactic analysis that integrates argumentation schemes with abstract argumentation. Schemes can be analysed into the roles that propositions play in each scheme and the structure of the associated propositions, yielding a greater understanding of the schemes, a uniform method of analysis, and a systematic means to relate one scheme to another. This analysis of the schemes helps to clarify what is needed to provide denotations of the terms and predicates in a semantic model.
\end{abstract}

Keywords: Abstract argumentation, instantiated argumentation, argumentation schemes, functional analysis

\section{Introduction}

Argumentation has proved useful in multi-agent systems (MAS) to represent dialogue (e.g. [33]), reasoning about action selection (e.g. [2,27]), and reasoning with inconsistent knowledge bases (KBs) $[9,24,25]$ in argumentation frameworks [23]. Argumentation schemes (AS) originated in informal logic (e.g. $[22,35])$ to represent arguments that are acceptable in ordinary conversation but are classical fallacies. A catalogue of schemes appears in [36]. They have also been used in computational argumentation to generate arguments and attacks on arguments, thus integrating ASs into abstract argumentation frameworks. For example, in one approach, schemes can be viewed as defeasible inference rules in a KB as in ASPIC+ [25]. A second approach refines the definition of a scheme by providing a semantic model, where the syntactic constituents of the AS have corresponding denotations in the model, e.g. the Practical Reasoning scheme in [5,6]; an instance of an AS is taken as an abstract Dungian argument, and arguments that attack it can be generated with reference to the model. A structural analysis of ASs also appears in [30]. Arguments generated using these approaches can be organised into argumentation frameworks, where arguments and attacks between them can be represented as nodes and arcs in directed graphs. Argumentation frameworks can be evaluated using a variety of semantics, such as grounded, preferred, and stable. A third approach relates ASs in ontological terms [28], which supports the annotation of texts. These approaches are related and address different parts of the computational analysis, use, and evaluation of ASs.

In this paper, we extend the second approach in a novel way by functionally tying together an abstract argument, the role of each proposition in an argumentation scheme, and the structure of the propositions. The result is a formal, fine-grained, and systematic analysis of argumentation schemes that feeds into abstract argumentation. The analysis is presented stepwise, starting from source data and proceeding 
to successively more abstract levels, along the way providing an intermediate level of representation for ASs. In this, the analysis systematically connects the source natural language expression of the argumentation scheme with its abstract argument; instantiated ASs provide arguments suitable for argumentation frameworks, while having an appropriate internal structure that can be used to capture important elements of the meaning of the original text. We establish a methodology for analysing ASs into their constituent parts that is suitable for computational modelling, in particular, the functional and Logic Programming paradigms. We use our analysis to relate schemes in virtue of common predicates and terms, where the conclusion of one scheme provides part of the justification in some other scheme. This offers a different perspective from the class-subclass relationships that arise naturally when building an ontology of schemes. In our view, it is key to have a detailed analysis of AS syntax in order to provide a basis for development of fine-grained semantic models and for generating attacks from those models. The paper contributes to the theory of MAS argumentation by providing an analysis of ASs that can be used in argumentation between agents.

The chief novel contributions of the paper are: the levels of analysis of ASs from natural language arguments to coarse-grained and fine-grained computational forms, the connection between the scheme structure and its constitutive propositions, and the specification of computational relationships between schemes. The analysis can also be used to understand some of the different formulations of ASs as in $[5,15,25,32]$, though we do not carry out such a comparative study here. From a larger perspective, the analysis begins to bridge the gap between the realisations of argumentation in natural language and their formal analysis. The analysis elaborates [5], where the ASs can be grounded in a semantic model, yielding benefits similar to those in model based diagnosis systems [16,31], where we can reason from first principles specific to the domain.

In Section 2, we discuss the levels of ASs, starting from a natural language instance of an AS and ending with abstract arguments. Initially, the analytic method is illustrated for the Position to Know AS. Section 3 formalises our analysis. The method is extended in Section 4 to various arguments about matters of fact, where we also discuss the relationships between schemes. In Section 5, we discuss the analysis, related work, and future directions.

\section{Levels of description in argumentation schemes}

Following [35], an AS is a stereotypical pattern of reasoning in which the premises give a presumptive reason to accept the conclusion. ASs are intended to be used in a dialogical context, where they are given as justifications for a conclusion and are subject to a critique covering a set of points characteristic of the particular scheme. An interlocutor might pose questions to elicit an answer that either contradicts, reaffirms, or otherwise weakens the rhetorical force of the AS. Consider, for example, the well-known Argument From Position to Know, where $E$ is an individual and $P$ a proposition; we will use this scheme as a running example.

- If $E$ is in a position to know $P$; and

- $E$ asserts $P$.

- Therefore $P$.

This is not a logically sound argument, but is widely used and accepted. Like any argument, this argument can be attacked by offering reasons to believe that its conclusion is false (rebuttal), by showing that 
Table 1

Levels of description of argumentation schemes

\begin{tabular}{llc}
\hline Level & \multicolumn{1}{c}{ Representation } & Reference \\
\hline $\mathbf{6}$ & Canonical sentences & {$[36]$} \\
$\mathbf{5}$ & Labelled roles and strings & {$[15,32]$} \\
$\mathbf{4}$ & Labelled roles and instantiated predicates & {$[4,5]$} \\
$\mathbf{3}$ & Functional roles and instantiated predicates & {$[25]$} \\
$\mathbf{2}$ & Functional roles and typed propositional functions & {$[4]$} \\
$\mathbf{1}$ & Atomic arguments & {$[23]$} \\
\hline
\end{tabular}

one of the premises is false (undermining), or by giving a reason to believe that the rule is inapplicable (undercut). ${ }^{1}$

To understand, interrelate, implement, and make ASs compatible with argumentation frameworks, we analyse them at different levels of representation. Hitherto, the lack of a formal analysis of the instantiated forms of the schemes as found in informal logic, such as [36], has given rise to confusion, especially in relation to abstract argumentation. This has proven a barrier to the meaningful use of ASs in computational models. To clarify these matters, we introduce a method of analysis that yields several related levels of representation from natural language expressions to fully abstract arguments, where the scheme level sits in the middle. Table 1 indicates the representation and an indicative, relevant reference. Each level is exemplified and discussed in Section 2.1. Our main, novel proposal is an articulation of Level 2, which in our view is the appropriate level to refer to an AS that relates to an argumentation framework.

There are three related objectives of the analysis. First, we want to relate ASs in natural language to arguments that can then be evaluated in abstract argumentation frameworks, providing a method that can be applied to a variety of ASs. Second, we want to analyse the characteristic components of a particular AS, giving the roles of the propositions in the AS and the associated internal structure of the propositions. Third, we want to relate ASs one to the other in light of their characteristic components. In this section, we address the first two points. The third point is discussed further in Section 4. We illustrate the method with our running example scheme, moving from a concrete example to successively more analytic representations (from Sixth to First levels in Table 1). Underlying the manner of presentation is the assumption that our analysis in this section proceeds by analysing fragments, where a fragment is a subset of the language for which we develop a syntax and semantics [21]; that is, we do not attempt to provide an analysis for all ASs of the "whole" language, which is indeterminate. Each AS uses its own fragment; as we understand additional ASs, we extend the analysis.

What counts as an AS or as different ASs is not clear in the literature, where the same AS can be seen in various formulations, and different catalogues of schemes are given (a point further discussed in Section 4). We presume canonical forms, selected from the range of synonymous lexical and syntactic variants, while acknowledging this is subject to further research. For our running example, we have the following, where the presumptive conclusion follows from the conjunction of the two premises.

Level 6 - Canonical sentences.

- Ms. Peters is in a position to know whether Mr. Jones was at the party.

- Ms. Peters asserts that Mr. Jones was at the party.

- Therefore, presumptively, Mr. Jones was at the party.

\footnotetext{
${ }^{1}$ There is no terminological consensus about the 'parts' of arguments. For our purposes, we follow the terminology in [25].
} 


\subsection{From argumentation scheme data to abstract arguments}

Starting with the AS data in natural language of Level 6, we incrementally decompose it into its formal, constitutent parts and their relationships, showing the method not only provides the result for this particular scheme, but also shows how to analyse other ASs in a systematic manner. In Section 3, we provide our formal language of ASs.

From the data, we identify: the terms and predicates of the argument's propositions, and the roles that propositions play in the AS. We use these to draw out some guiding intuitions. The terms and predicates could be expressed in a suitable logic of the associated expressions and represented in a KB. As for roles, there appear to be two distinct sorts that propositions play in ASs - scheme generic and scheme specific. The scheme generic roles associate propositions with the logical role in the argument irrespective of the propositional content, much as the logical connectives apply to any proposition; in contrast, scheme specific roles highlight aspects of domain information in the propositions, where specific schemes present specific content. There is a fixed and finite set of scheme generic roles such as are typically labelled Premise and Conclusion ${ }^{2}$ in principle, any proposition could fulfill these roles. The scheme specific roles identify the particular role in respect of the propositional content of the proposition that the roles apply to. For instance, with respect to our sample scheme, we have propositions about an individual being in a position to know and about the assertion that individual makes. The scheme requires two premises, one of each type, and linked in a specified way.

\subsubsection{Level 5}

These guiding intuitions indicate that it will be useful to represent the specific roles as modifiers of the generic roles, giving us a positionToKnow-Premise, an assertion-Premise, and a conclusion; such labelled roles appear in the appendix of [36], though not consistently nor with further analysis. More specifically, we subsort and label the premises for the role that the proposition plays in the argument. Interestingly, there is no related conception of differentiating the conclusion with respect to specific ASs; this is to do no more than to say that all ASs have a conclusion; we shall have reason to revise this view where we discuss Level 3 since plainly conclusions are tied to schemes.

\section{Level 5 - Labelled roles and strings.}

- positionToKnow-Premise: Ms. Peters is in a position to know whether Mr. Jones was at the party.

- asserts-Premise: Ms. Peters asserts that Mr. Jones was at the party.

- concludes: Mr. Jones was at the party.

The assumption here (and the other levels below) is that where the premises conjunctively hold, the conclusion presumptively follows.

\subsubsection{Level 4}

At Level 5, there is an implicit relationship between the labels and the content of the strings, which needs to be analytically explicit; after all, the positionToKnow-Premise ought not to label just any string.

\footnotetext{
${ }^{2}$ There may be alternatives, e.g. assumption, issue, and ordinary premise, as in [25], Definition 3.5, though they are not highly relevant for us at the moment. However, we note the point is a bit subtle. For example, whether a particular proposition is an exception or assumption or ordinary premise (to follow the terminology of [15]) is tied to the propositional content, the relation of the given proposition to the other propositions in the argument, or even on whether the speaker or the hearer has the burden of proving the proposition if it challenged; that is, even these generic roles are contextual in the argument, and as the context varies, so varies the role. The important point for our purposes is that the generic roles are not themselves labelled with respect to the domain terminology.
} 
To draw out this relationship, we represent the predicates and terms, helpfully reusing the predicate in the role label:

Level 4 - Labelled roles and instantiated predicates.

- positionToKnow-Premise: positionToKnow(Ms. Peters, Mr. Jones was at the party).

- asserts-Premise: asserts(Ms. Peters, Mr. Jones was at the party).

- concludes: Mr. Jones was at the party.

In other words, we have not only labelled strings, but we have begun to formalise the meaning of the string in a correlated logic-like language.

However, before further analytic steps, we add to our representation premises for rules. In our view, it is useful to be able to differentiate arguments concerning acceptance of the rule as it is from those concerning normative circumstances for the application of the rule. In general, arguments have a structure idealised as Modus Ponens of Classical Propositional Logic, where there are premises that are literals as well as a rule that has the literals in the antecedent of the rule and another literal in the conclusion; where the literals and rule hold, one infers that the conclusion of the rule holds. There are strict and defeasible rules, symbolically indicated with $\rightarrow$ and $\rightsquigarrow$ respectively. However, while Modus Ponens of Classical Propositional Logic must explicitly provide the rule, in arguments as they commonly appear, rules seem rarely to be stated explicitly as a premise of the argument: if someone says $Q$ since $P$, this introduces the presupposition that the speaker has committed to $\mathrm{P}$ as well as to a rule if $P$, then $Q$. As ASs are presumptive, they would include a defeasible rule. We believe defeasible rules in ASs appear under two "guises" - the rule itself and a rule with respect to normative circumstances; for the former, we can argue whether or not we accept the rule, while for the latter we can argue that though we accept the rule, the circumstances are not normative for its application. For our purposes in formalising ASs, it is important to explicitly express the rule as we can then allow two different ways to attack an AS with respect to a rule.

For a rule that is accepted, we can simply state the rule. For a normative application of a rule, we believe that a theory of circumscription $[19,20]$ is useful. Broadly, circumscription presumes that the situation is as normal or regular as we understand it ought to be, given our knowledge. It addresses the frame problem by bounding explicit conditions to only those generally understood to be relevant to solving a problem and excluding irrelevant exceptional circumstances; it handles inertia by assuming that unless explicitly changed by an action, all else remains constant. Of course, for reasoning about different circumstances and actions, there are distinct relevant conditions or consequences of actions. Thus, some representation of such variety is needed.

To make relativised circumscription explicit, we use of a designated predicate $a b C i r c$ applied to a rule $r_{i}, a b \operatorname{Circ}\left(r_{i}\right)$, which means the circumstances are abnormal for the application of the rule $r_{i}$. We introduce $\neg a b \operatorname{Circ}\left(r_{i}\right)$ as a negated premise (an exception), which we label ceteris paribus, meaning "all other things being equal"; a rule applies unless the circumstances are abnormal. ${ }^{3}$ Where $\operatorname{abC\operatorname {Circ}}\left(r_{i}\right)$ holds, the circumstances are abnormal and ceteris paribus is perturbed, so we cannot reason to the conclusion even though the rule holds; we have undercut the applicability of the rule. Where $\neg a b \operatorname{Circ}\left(r_{i}\right)$ holds, the circumstances fulfill ceteris paribus, so we can reason to the conclusion using the rule, assuming the premises of the rule also hold. While we can give a list of sufficient conditions for abCirc to hold, this list will never, even in principle, be complete. On the other hand, we may have a circumstance which fulfills ceteris paribus, yet, it is argued that the rule itself is not correct; here, we have undermined the

\footnotetext{
${ }^{3}$ Ceteris paribus is used in the physical and social sciences to hold factors constant for the application of the rule.
} 
argument by denying the rule premise. The $a b$ Circ predicate and rule appear in our running example as follows, which is still Level 4, but with the auxiliary information about the rule and the abCirc predicate:

Level 4 - Labelled roles and instantiated predicates with abCirc and rule.

- positionToKnow-Premise: positionToKnow(Ms. Peters, Mr. Jones was at the party).

- asserts-Premise: asserts(Ms. Peters, Mr. Jones was at the party).

- ceterisParibus-Premise: $\neg$ abCirc([positionToKnow(Ms. Peters, Mr. Jones was at the party) $\wedge$ asserts(Ms. Peters, Mr. Jones was at the party)] $\rightsquigarrow$ Mr. Jones was at the party).

- rule-Premise: [positionToKnow(Ms. Peters, Mr. Jones was at the party) $\wedge$ asserts(Ms. Peters, Mr. Jones was at the party)] $\rightsquigarrow$ Mr. Jones was at the party.

- concludes: Mr. Jones was at the party.

The ceteris paribus premise is easily recovered from the rule, so can be suppressed. Literals and the rule are taken from the KB to construct the AS; consequently, given the AS itself with the premises and conclusion, we can recover the rule, so that we can suppress it as well.

\subsubsection{Level 3}

To this point, we have a logic-like expression of an AS. One of our chief goals is to relate ASs to argumentation frameworks, which requires that we associate ASs with abstract arguments. One approach to this association is the ASPIC approach to argumentation, best expressed in [25] and referred to as ASPIC +. We will draw comparisons to ASPIC + in the remainder of this paper, noting where we deviate from it, e.g. circumscription deviates from [25], Definition 3.4. We turn to [25], Definition 3.6 and dependent definitions, where arguments are defined syntactically and generic roles are functions from arguments to propositions from the KB. There are several parts to the definition that are relevant to our purpose - arguments, rules, and premises/conclusions. Our analysis proceeds in two analytic steps, first introducing functions from arguments to expressions of a KB (along the lines of [25]), then introducing propositional functions, which abstract from the propositions of Level 4.

In [25], arguments are syntactic abstract objects in the sense that they are constructed from expressions in the KB. These objects are then are used in an argumentation framework. In contrast, we do not provide a syntactic definition of arguments, but of ASs; arguments themselves are semantic abstract objects - individuals in the semantic model to which we can apply functions and which have attributes. ${ }^{4}$ Furthermore, while arguments may be atomic or complex (having subarguments), we consider for our purposes arguments without subarguments (though we return to this topic later).

Turning to the specific structure of an argument in [25], Definition 3.6, the elements of the arguments (premise, conclusion, and rule) are not just listed informally (as in Levels 4 and 5), but are functions from arguments. We assume a $\mathrm{KB}$, which in [25], is expressed in a well defined language and subject to some (unspecified) logic. The Prem function is from arguments to the set of literals from the KB, giving the premises; the function Conc is from arguments to a literal from the KB, giving the conclusion.

In ASPIC+, premises are homogenised rather than differentiated as in Levels 4 and 5. To give more fine-grained semantic information as required for ASs, we subsort the premise function into several premise functions (and keep a function for the conclusion); thus, the premises are heterogeneous. Furthermore, we want to explicitly associate the premises with the predicates and terms of the correlated expression; to do this, we prefix the premise function with the predicate of the associated expression. In addition, as we are considering the structure of ASs and not just some particular instance, we want

\footnotetext{
${ }^{4}$ Arguments have the attributes of abstract objects: they do not "exist" as objects that have physical attributes, yet they can be quantified over (every argument), referred to (that argument), and bear properties (an attractive argument) [3].
} 
the level of representation where the expressions have not yet been grounded. The justification for subsorting the premises is that literals play different and restricted roles in an AS, and the justification for particular premises of an AS may depend on the literal being justified. These distinctions are not made explicit in a homogeneous structure for arguments. For instance, to define the Position to Know AS, there must be one proposition that presents the position to know, one proposition that presents the assertion, and (aside from the rule and the abCirc predicate) no other premises. Moreover, the justification for the positionToKnow-Premise is different from the justification of the asserts-Premise. Turning to the concludes function, we find that it is important to clearly differentiate the sorts of the conclusions of arguments (particularly where the rule is suppressed): the conclusion of an AS is semantically particular to that AS, e.g. the meaning of the Position to Know AS conclusion is systematically different from the meaning of the conclusion of the Practical Reasoning AS, and so on for other ASs. Furthermore, where ASs are combined and unified, a point we illustrate in Section 4, it is technically important to differentiate conclusions. Therefore, we assume that the conclusion function is differentiated with respect to the AS; in other words, a conclusion of an AS is a conclusion of that AS and not of another AS.

Following this analysis, we have functions applied to an argument $a_{i}$ :

Level 3 - Functional roles and instantiated predicates.

- positionToKnow-PremiseF $\left(a_{i}\right)=$ positionToKnow(Ms. Peters, Mr. Jones was at the party).

- asserts-PremiseF $\left(a_{i}\right)=$ asserts(Ms. Peters, Mr. Jones was at the party).

- ceterisParibus-PremiseF $\left(a_{i}\right)=\neg$ abCirc([positionToKnow(Ms. Peters, Mr. Jones was at the party) $\wedge$ asserts(Ms. Peters, Mr. Jones was at the party)] $\rightsquigarrow$ Mr. Jones was at the party).

- rule-PremiseF $\left(a_{i}\right)=$ [positionToKnow(Ms. Peters, Mr. Jones was at the party) $\wedge$ asserts(Ms. Peters, Mr. Jones was at the party)] $\rightsquigarrow$ Mr. Jones was at the party.

- positionToKnow-Concludes $F\left(a_{i}\right)=M r$. Jones was at the party.

\subsubsection{Level 2}

However, the previous levels are not, in our view, truely schemes since they are all fully instantiated, that is, we are using predicates and terms that are constants rather than predicates and variables. To represent a scheme as such, we must abstract over the instantiated expressions. Here we present the formalisation of the working AS; in Section 3, we provide a general, formal language of ASs. As predicates are key to our analysis of ASs, we suppose a syntactically typed and semantically sorted language for the expressions of the KB. For example, $X_{\text {argument }}$ is a variable subtyped for argument, $Y_{\text {person }}$ is a variable subtyped with respect to person, while $Y_{\text {literal }}$ is a variable subtyped with respect to literal (an atomic proposition, which bears a truth value, or its negation). The variables for literals are a convenience since they could be analysed as predicates applied to terms, but this requires a more extensive analysis than is needed for our purposes. We assume that predicates are also typed and sorted with respect to the types and sorts of their terms (but suppress this in the presentation for readability). The syntactic terms and predicates have denotations in corresponding semantic sorts. As we assume only finitely many sorts, we could instead use more verbose expressions in an unsorted first order language with unary predicates that partition the domain of discourse. For instance, rather than $Y_{\text {person }}$ and $V_{\text {book }}$, we could instead have predicates such as person $(x)$ and $\operatorname{book}(x)$, where no individual is both a person and book. By the same token, Logic Programming is untyped, but can achieve the effect of typing with special, defined predicates. Broadly speaking, the predicate and term types simplify the discussion; a richer discussion requires, among other things, representations for intensional "objects" such as the propositions that are the complements of propositional attitudes. We refer to propositions with free variables as propositional functions since they are functions from individuals (or ordered n-tuples of individuals) to truth values; the 
propositional function would, when instantiated, represent a literal or a relation between an individual and a literal.

Turning to the AS functions, we have premise functions associated with predicates, indicated as $\mathcal{X}$-Premise $\mathcal{F}$, from variables of type $X_{\text {argument }}$ to the corresponding propositional function, where $\mathcal{X}$ is a string that matches the predicate of the propositional function. We have a concludes function $\mathcal{Y}$-concludes $\mathcal{F}$, where the $\mathcal{Y}$ string is the label for the AS; in our working AS, $\mathcal{Y}$-Concludes $\mathcal{F}$ is a function from variables of type $X_{\text {argument }}$ to a variable of type $Z_{\text {literal }}$. For example, where the premise function is positionToKnow-Premise $F\left(X_{\text {argument }}\right)$, the output is positionToKnow $\left(Y_{\text {person }}, Z_{\text {literal }}\right)$; for positionToKnow-Concludes $F\left(X_{\text {argument }}\right)$, the output is $Z_{\text {literal }}$.

Finally, the ceterisParibus-Premise and rule-Premise are constructed with respect to the other premise functions. The ceterisParibus-Premise functions are from variables of type $X_{\text {argument }}$ to expressions of type $a b C i r c$, which are propositional functions of the form $\neg$ abCirc (rule-Premise $\left.F\left(X_{\text {argument }}\right)\right)$; this is well formed when the argument is instantiated. The rule-Premise function is from variables of type $X_{\text {argument }}$ to expressions of type Rule. In turn, expressions of type Rule have a specific structure - the body of the rule is the set of premise functions associated with predicates (above), and the head of the rule (the conclusion) is a literal from the KB. ${ }^{5}$ The formal definitions for both these sorts of premise functions are given in Section 3.

We can now represent our working example AS formally in a language compatible with formal argumentation, where the instantiation of $X_{\text {argument }}$ is an abstract argument in an argumentation framework. Yet, the important point is that our schematic representation connects the argument with the propositional functions that, given suitable grounding, represent the semantic content of the scheme.

\section{Level 2 - Functional roles and typed propositional functions.}

- positionToKnow-PremiseF $\left(X_{\text {argument }}\right)=\operatorname{positionToKnow~}\left(Y_{\text {person }}, Z_{\text {literal }}\right)$.

- asserts-Premise $F\left(X_{\text {argument }}\right)=\operatorname{asserts}\left(Y_{\text {person }}, Z_{\text {literal }}\right)$.

- ceterisParibus-Premise $F\left(X_{\text {argument }}\right)=\neg a b \operatorname{Circ}\left(\right.$ rule-Premise $\left.F\left(X_{\text {argument }}\right)\right)$.

- rule-Premise $F\left(X_{\text {argument }}\right)=\left[\right.$ positionToKnow $\left.\left(Y_{\text {person }}, Z_{\text {literal }}\right) \wedge \operatorname{asserts}\left(Y_{\text {person }}, Z_{\text {literal }}\right)\right] \rightsquigarrow Z_{\text {literal }}$.

- positionToKnow-Concludes $F\left(X_{\text {argument }}\right)=Z_{\text {literal }}$.

As in Prolog clauses, variables must be consistently instantiated within a scheme. At this level of analysis, we have term variables and propositional functions, yet the scheme functions and the predicates are instantiated. ${ }^{6}$ In our view, this is the appropriate level to bear the title argumentation scheme as such since, properly speaking, a scheme is only a scheme when it has uninstantiated term variables for the propositional functions. Yet, the conceptual content of the particular AS is represented by the specific propositional functions.

Before discussing the next level, we digress. One might propose another abstract scheme where we have an unsorted premise function, which is conceptually equivalent to the premise function as found in ASPIC+ [25], Definition 3.6. Here the output of the function conj-Premise is not a literal or a relation, but a conjunction of literals; the output of conj-Concludes is a literal from among the conjoined literals;

\footnotetext{
${ }^{5}$ In argumentation, the rule may be attacked either with respect to abnormal circumstances or the rule itself; thus the negation of a rule or an abCirc predicate may appear as the conclusion of an argument. While such arguments can be formalised in ASPIC + [25], our formalisation does not characterise them as argumentation schemes, which are prototypical, presumptive patterns of reasoning, as we are not aware of such patterns where the conclusion is the negation of a rule or an abCirc predicate. Should it demonstrated otherwise, our formalisation would change accordingly.

${ }^{6} \mathrm{We}$ could abstract over the predicates and functions as well given a second order language.
} 
the rule is strict, and there is no abCirc predicate. As a whole, the argument has the form of Conjunction Reduction in Propositional Logic; it is unclear to us what such a scheme means where the rule is applicable ceteris paribus since in principle for such a scheme there cannot be any relevant variation in context.

Conjunction reduction.

- $\operatorname{conj-Premise} F\left(X_{\text {argument }}\right)=X_{\text {literal }}, \wedge, \ldots, \wedge Z_{\text {literal }}$.

- $\operatorname{rule-Premise} F\left(X_{\text {argument }}\right)=\left[X_{\text {literal }}, \wedge, \ldots, \wedge Z_{\text {literal }}\right] \rightarrow \operatorname{conj-Concludes} F\left(X_{\text {argument }}\right)$.

- $\operatorname{conj-Concludes} F\left(X_{\text {argument }}\right)=Y_{\text {literal }}$.

While one might take this as a "generic" AS, we propose that it is something different from the AS we find at Level 2 in three respects. First, there are no functions that depend on the semantic content of the literals. Second, it is not an abstract representation of our presumptive, defeasible ASs since conjunction reduction is strict. Third, other ASs have conjunctive premises (though differentiated) and the premise function of Conjunction Reduction is not associated with any semantic content of the literals. For these reasons, we prefer to only call structures in the form of Level 2 an AS and not structures of the form for Conjunction Reduction or other standard inference rules of Propositional and Predicate Logic, which we refer to as Logical Schemata. It may be that there are ASs that have a representation where the premises are unsorted, but this remains an open question for us.

Conjunction Reduction should not be conflated with an AS that has a conjunction of literals. For instance, in an analysis of the Value-based Practical Reasoning AS [5], it is proposed that there are premises that represent the current circumstances and consequences of actions, each of which denote a state that can be specified as a conjunction of literals. As with other ASs, such a scheme requires some extension to the fragment currently under discussion, in particular to express circumstances, consequences, actions, and values. This seems straightfoward, as actions and values type variables, and we can allow circumstance and consequence predicates on a conjunction of literals; we have the associated functions on arguments. Because of this, we can say such a scheme is represented in the manner of Level 2.

\subsubsection{Level 1}

Returning from the digression, we have Level 1 where we have argument individuals, $\left\{a_{0}, \ldots, a_{n}\right\}$, that ground ASs at Level 2, associating these individuals with properties. Argument individuals are the objects which can be used (given attacks as specified in Section 3) for evaluation in an argumentation framework as in [23] and subsequent work. Given indexed argument individuals and the functional definition of argumentation schemes, we can always recover (by the inverted function) the semantic content that the argument is related to; in this respect, we can identify such an argument with the instantiated AS in which it appears, for the AS may be taken as the characterisation or internal structure of the argument. In this respect our analysis differs from abstract argumentation, where arguments as [23] are not associated with any AS, have no internal structure, and cannot justify any conclusion nor justify attacks since the internal structure of the arguments is not available. Rather abstract arguments are used in the evaluation of sets of arguments. In contrast, justifications are a primary motivation for our analysis of ASs. On the other hand, our analysis does not introduce undue complexity into abstract argumentation itself, in which regard our analysis serves as a systematic bridge between abstract and instantiated argumentation.

To end this section, it is important to emphasise again that the language we have outlined above is an indicative specification for the fragment, not a formal specification of this or all ASs since the range of alternative interpretations or possible expressions remains to be determined. More broadly, it remains 
to be investigated how expressive the language of arguments must be to accommodate the expressive range of linguistic forms. But, we have already indicated how this might be done for one AS, and we will see how this can be done for other ASs in Section 4. As well, there may be reason to further analyse the Position to Know AS; for instance, properly speaking, the "object" of the predicate "know" is better represented as a proposition in an intensional semantics, yet to provide this analysis opens the door to a fuller formal analysis of natural language syntax and semantics [13], which while relevant, detracts from the focus in this paper on the formal analysis of ASs. On the other hand, we assume that the premise functions are as fine-grained as their corresponding propositional functions.

\section{A functional language for argumentation schemes}

In this section, we present our formal language for ASs. We have a language, specifications for ASs, identity conditions, and a definition of the attack relation. We assume a logical language $\mathcal{L}$, a finite language of syntactically typed and semantically sorted predicates, terms, and variables. We have illustrated a fragment of such a language above and leave further specification open-ended and extensible since it depends not just on what schemes we want to represent, but also on further analysis of the schemes we have. We assume classical negation $\neg .{ }^{7}$ There are expressions of type Rule that are strict $\rightarrow$ or defeasible $\rightsquigarrow$; the bodies are conjoined expressions of $\mathcal{L}$, and the heads are propositions of $\mathcal{L}$. As a subset of the variables, we have types and sorts for arguments and literals. We also have a designated predicate abCirc that applies to expressions of type Rule. Finally, we have a finite set of AS labels, $\mathcal{G}{ }^{8}$ With respect to the language, we have component definitions.

\section{Definition 1 (AS Functions).}

1. A set of functional premises $\mathcal{D}$, where the functions have the form: $\mathcal{X}^{\prime}$-Premise $F\left(X_{\text {argument }}\right)$ and are from variables of type $X_{\text {argument }}$ to the propositional function where the predicate is $\mathcal{X} . \mathcal{X}^{\prime}$ is an element of $\mathcal{G}, \mathcal{X}$ is an element of the predicates of $\mathcal{L}$, and $\mathcal{X}^{\prime}$ is functionally derived from $\mathcal{X}$.

2. A set of functional ceterisParibus premises $\mathcal{N}$, where the functions have the form: ceterisParibus-Premise $F\left(X_{\text {argument }}\right.$ ) and are from variables of type $X_{\text {argument }}$ to a propositional function of the form $\neg a b \operatorname{Circ}(\mathcal{Z})$, where $\mathcal{Z}$ is of type Rule and the rule connective is $\rightsquigarrow$.

3. A set of functional rule premises $\mathcal{R}$, where the functions have the form: rule-Premise $F\left(X_{\text {argument }}\right)$ and are from variables of type $X_{\text {argument }}$ to an expression of type Rule and the rule connective is $\rightsquigarrow$.

4. A set of functional conclusions $\mathcal{C}$, where the functions have the form:

$\mathcal{Y}$-Concludes $F\left(X_{\text {argument }}\right)$ and are from variables of type $X_{\text {argument }}$ to a literal $\mathcal{Z}$, and $\mathcal{Y}$ is an element of $\mathcal{G}$.

Definition 1 formalises the parts of ASs, where the Level 2 representation is an example; the functions on arguments are defined with respect to the content of the correlated propositions. Definition 1 (1.) provides the non-rule premises of the AS, associating the particular label of the premise to the propositional function; Definition 1 (2.-3.) are the rules of the AS; Definition 1 (4.) is the conclusion. The functions

\footnotetext{
${ }^{7}$ Though see discussion in [25] on a contrariness function.

${ }^{8}$ We suppress a one-to-one function from predicates to correlated labels, which are strings that can serve as prefixes to the premise and conclusion function.
} 
define argumentation schemes, not more general patterns of reasoning that also serve as arguments and are definable in terms of ASPIC+ [25] such as Conjunction Reduction discussed above. In particular, arguments with a conclusion that is the negation of a ceterisParibus or a rule premise are not elements of $\mathcal{C} .{ }^{9}$

Definition 2 (Function distribution).

1. There is a non-empty set $\mathcal{D}_{A S_{i}} \subset \mathcal{D}$; and

2. There is exactly one function $\mathcal{N}_{A S_{i}} \in \mathcal{N}$; and

3. There is exactly one function $\mathcal{R}_{A S_{i}} \in \mathcal{R}$; and

4. There is exactly one function $\mathcal{C}_{A S_{i}} \in \mathcal{C}$.

Definition 3 (Function constraints).

1. For $\mathcal{N}_{A S_{i}}$ of form ceterisParibus-PremiseF $\left(X_{\text {argument }}\right)$, such that

$$
\text { ceterisParibus-PremiseF }\left(X_{\text {argument }}\right)=\neg \operatorname{abC\operatorname {Circ}}\left(\mathcal{R}_{A S_{i}}\right)
$$

and

2. For $\mathcal{R}_{A S_{i}}$, where the output expression is a rule with body $\mathcal{B}$ and head $\mathcal{H}, \mathcal{B}=\mathcal{D}_{A S_{i}}$ (the set interpreted conjunctively), and $\mathcal{H} \in \mathcal{L}$; and

3. $\mathcal{C}_{A S_{i}}=\mathcal{H}$.

Definition 2 constrains the numbers of functions for a particular AS, while Definition 3 ties the ceterisParibus premise to the rule and the rule to the other premises and conclusion of the AS.

Definition 4 (Definition of AS well-formedness). An $A S_{i}$ is well-formed if and only if the functions are given as in Definitions 1-3, and $A S_{i}=\mathcal{D}_{A S_{i}} \cup\left\{\mathcal{N}_{A S_{i}}\right\} \cup\left\{\mathcal{R}_{A S_{i}}\right\} \cup\left\{\mathcal{C}_{A S_{i}}\right\}$.

Definition 4 specifies that an AS is well-formed if and only if it has all the relevant "parts" of an AS.

Definition 5 (AS identity conditions). $A S_{i}$ and $A S_{j}$ are identical if and only if $A S_{i}=A S_{j}$, otherwise $A S_{i} \neq A S_{j}$.

Given our set-theoretic definition of an AS, Definition 5 for the identity condition is such that any two ASs are identical if and only if they have all the same "parts". This is a rather stringent constraint since, after all, different wording or syntactic structures might be used in two natural language ASs, but which are taken to be synonymous. These are general, well-known issues of lexical semantic and syntactic analysis, e.g. when are two sentences related by synonymy, contradiction, or entailment [11]. Other relationships between schemes can be defined set-theoretically, e.g. subarguments. Both topics are touched on in Section 4.

Finally, we turn to the various notions of attack, where one argument attacks another with respect to specific "parts" of an AS.

\footnotetext{
${ }^{9}$ The rationale for this constraint on well-formed argumentation schemes is empirical - we know of no proposal for patterns of presumptive, defeasible reasoning for ceterisParibus or a rule statements; where such examples to be provided, the definition of $\mathcal{C}$ would be generalised.
} 
Definition 6 (Argument attack). $X_{\text {argument }}$ attacks $Y_{\text {argument }}, X_{\text {argument }} \neq Y_{\text {argument }}$, if and only if $X_{\text {argument }}$ rebuts $Y_{\text {argument }}$, or $X_{\text {argument }}$ undermines $Y_{\text {argument }}$, or $X_{\text {argument }}$ undercuts $Y_{\text {argument }}{ }^{10}$

Definition 7 (Rebuttal, Undermining, and Undercutting).

1. $X_{\text {argument }}$ rebuts $Y_{\text {argument }}$ if and only if

$$
\mathcal{X} \text {-Concludes } F\left(X_{\text {argument }}\right)=\neg\left(\mathcal{Y} \text {-Concludes } F\left(Y_{\text {argument }}\right)\right) .
$$

2. $X_{\text {argument }}$ undermines $Y_{\text {argument }}$ if and only if

- $\mathcal{X}$-Concludes $F\left(X_{\text {argument }}\right)=\neg\left(\mathcal{Z}\right.$-Premise $\left.F\left(Y_{\text {argument }}\right)\right)$, for some premise prefix $\mathcal{Z}$; or

- $\mathcal{X}$-Concludes $F\left(X_{\text {argument }}\right)=\neg\left(\right.$ rule $\left.-\operatorname{Premise} F\left(Y_{\text {argument }}\right)\right)$.

3. $X_{\text {argument }}$ undercuts $Y_{\text {argument }}$ if and only if

$$
\mathcal{X} \text {-Concludes } F\left(X_{\text {argument }}\right)=\neg\left(\text { ceterisParibus-Premise } F\left(Y_{\text {argument }}\right)\right) .
$$

These notions of attack are closest to the basic attack conceptions of [23,25], not to richer notions of defeat, where attacks are relativised to preferences or values $[1,8]$, though one might incorporate them. It is worth emphasising (following $[9,25]$ ) that arguments and attacks on arguments are entirely constructed from information found in the (inconsistent) knowledge base, meaning that there are no ad $h o c$ attacks. At this point, we can turn attention to the structure of a set of closely related schemes.

\section{Relating schemes}

Having introduced a method and a formalisation illustrated with a worked example, we consider richer schemes and their relationships. A compendium of ASs is provided in [36]. Assuming that all schemes are represented in (or translated to) our formalisation of ASs, an AS is specified by its functions as in Definition 4; relationships between ASs such as identity and subset can be expressed in set theoretic terms. We discuss a family of schemes for arguing about facts to illustrate these relationships.

In 4.1, we discuss two ways to analyse ASs about facts. First, we take several schemes catalogued in [36] and express them in our formalisation, showing several limitations. Then, because of the limitations, we propose a reanalysis of the schemes into a main scheme Credible Source (also in [36]) with subsidiary ASs for particular premises, which are novel and not found in the literature, yielding a 'treelike' structure of related ASs (cf. [18]). Thus, unlike the compendium of ASs as in [36], we provide some decomposition and structure to ASs and their relationships. The analysis shows the strengths of our approach, for it helps to clarify fundamental issues about particular analyses of ASs, which can lead to improved ASs, and additional definitions on ASs.

\footnotetext{
${ }^{10}$ This encodes the conception that no instantiated argumentation scheme (and so no argument) can attack itself, which could only be the case were the conclusion of an argument to contradict a premise, rule, or abCirc of the same argument. Were such contradictions to arise within an argument, then any conclusion could be drawn. This follows the widespread conception in instantiated argumentation, e.g. [9,25], that arguments are internally consistent and that inconsistency only arises between distinct arguments.
} 


\subsection{Arguing about facts}

In [36], several related ASs are reported to establish matters of fact:

1. Argument from Position to Know (PK): Source $S$ is in a position to know about things in subject domain $\mathrm{D}$ containing proposition $\mathrm{P}$; and $\mathrm{S}$ asserts that $\mathrm{P}$ is true (false). Therefore, $\mathrm{P}$ is true (false).

2. Argument from Expert Opinion (EO): Source $E$ is an expert in subject domain D containing proposition $\mathrm{P}$; and $\mathrm{E}$ asserts that proposition $\mathrm{P}$ is true (false). Therefore, $\mathrm{P}$ is true (false).

3. Argument from Witness Testimony (WT): Witness W is in position to know whether $P$ is true or not; Witness W is telling the truth (as W knows it); and Witness W states that P is true (false). Therefore, P may be plausibly taken to be true (false).

4. Argument from Perception (P): Person P has an image I (an image of a perceptible property); and to have an image I (an image of a perceptible property) is a prima facie reason to believe that the circumstances $\mathrm{C}$ exemplify I. Therefore, it is reasonable to believe that $\mathrm{C}$ is the case. ${ }^{11}$

There appear to be two directions to take the analysis, and we illustrate them each to highlight how our approach shows issues concerning AS analysis. In one way of analysis, the direct analysis, we take the ASs essentially as they are, represent their logical forms, and then relate them. In the second approach, reanalysis, we reanalyse the variant schemes into essentially a main argument, here Credible Source, with subsidiary arguments for premises of the main scheme.

\subsection{Direct analysis}

The schemes PK, EO, WT, and P are clearly related, yet there are a range of variations which are not clearly relevant to the argument, and the schemes should be normalised and canonicalised to support formal integration (leaving standardisation open for future research). For example, all the conclusions are presumptive statements that some state of affairs holds. Yet, this only appears in WT. As well, we assume the arguments are always about reasonable belief. In other schemes there is variation such as asserts that $A$, asserts that proposition A, and states that. Our analysis forces out such incidental variation. For our purposes, we normalise the schemes and present them as ASs in our formalisation; some complex predicates in the data are decomposed in the analysis; propositions are presented as expressions of type $Z_{\text {literal }}$. Consistent with our assumptions above, the conclusion functions are all labelled according to the AS in which they appear, thus relativising the conclusion to the scheme in which it appears.

Position to Know (PK).

- positionToKnow-Premise $F\left(X_{\text {argument }}\right)=$ positionToKnow $\left(Y_{\text {person }}, Z_{\text {literal }}\right)$

- asserts-Premise $F\left(X_{\text {argument }}\right)=$ asserts $\left(Y_{\text {person }}, Z_{\text {literal }}\right)$

- positionToKnow-Concludes $F\left(X_{\text {argument }}\right)=Z_{\text {literal }}$

Expert Opinion (EO).

- expertAbout-Premise $F\left(X_{\text {argument }}\right)=\operatorname{expertAbout}\left(Y_{\text {expert }}, Z_{\text {domain }}\right)$

- inDomain-Premise $F\left(X_{\text {argument }}\right)=\operatorname{inDomain}\left(X_{\text {literal }}, Z_{\text {domain }}\right)$

- asserts-Premise $F\left(X_{\text {argument }}\right)=\operatorname{asserts}\left(Y_{\text {expert }}, X_{\text {literal }}\right)$

- expertOpinionAS-Concludes $F\left(X_{\text {argument }}\right)=X_{\text {literal }}$

\footnotetext{
${ }^{11}$ In [36, p. 345], it is claimed that what is reasonable to believe is I; however, the argument is not about the image since we cannot argue about whether or not an individual has an image, which is a question for epistemology rather than argumentation, but we can argue about whether or not the report of having the image in an argument for circumstances being one way or another.
} 
Witness Testimony (WT).

- positionToKnow-Premise $\left(X_{\text {argument }}\right)=$ positionToKnow $\left(Y_{\text {witness }}, Z_{\text {literal }}\right)$

- truthTelling-Premise $F\left(X_{\text {argument }}\right)=\operatorname{truthTelling}\left(Y_{\text {witness }}\right)$

- asserts-Premise $F\left(X_{\text {argument }}\right)=$ asserts $\left(Y_{\text {witness }}, X_{\text {literal }}\right)$

- witnessTestimony-Concludes $F\left(X_{\text {argument }}\right)=X_{\text {literal }}$

Perception (P).

- perceives-Premise $F\left(X_{\text {argument }}\right)=\operatorname{perceives}\left(Y_{\text {person }}, Z_{\text {percept }}\right)$

- perceptSupports-Premise $F\left(X_{\text {argument }}\right)=\operatorname{perceptSupports}\left(Z_{\text {percept }}, V_{\text {literal }}\right)$

- perception-Concludes $F\left(X_{\text {argument }}\right)=V_{\text {literal }}$

A range of issues arise about particular analyses, variations in the terms and predicates, and attendant relationships between the schemes. We see that the ASs intersect on some functions and not on others: PK and WT have positionToKnow; PK, EO, and WT have asserts; only P has perceptSupports. Each AS has a different conclusion function, which means that the literal that concludes an instantiated AS is particular (though not necessarily unique) to each AS.

A deeper level of observations touch on the issue of the fine-grained semantic analysis of the statements of the AS. To give one example, we see that several ASs have the function asserts-Premise $F\left(X_{\text {argument }}\right)$, which given our functional assumptions, ought to map to the same propositional functions. Yet, clearly they do not; in particular, we have predicates such as asserts applying to terms of different sorts such as person, expert, and witness. This might be taken to imply that either we have predicates similarly subsorted, to distinguish schemes, or we have polymorphic types.

One possible solution would be simply to abstract over the predicate asserts that varies and define its possible scope, e.g. $\mathcal{R}=$ \{expert', witness', person' $\}$, which essentially represents the set of sets of individuals that play a part in such ASs; the logical form for the assertion propositional function would have this variable in place of $\operatorname{expert}^{\prime}(x)$. In effect, the particular ASs are tied to a higher level abstract AS that comes with an associated ontology.

While we might treat the asserts-Premise in a relatively homogeneous manner, others are more problematic. On the face of it, there seems to be some semantic relationship between positionToKnow, expertAbout, and (perhaps) perceives. Yet, unless this relationship is further specified (as we have done with respect to asserts), the ASs are otherwise unrelated. A similar point applies to asserts and perceptSupports.

Unless and until these lexical semantic relationships between terms and predicates are resolved, we cannot say much more about the relationships between the associated ASs. The discussion is instructive in any case, for by formally clarifying the space of issues, we can systematically address them in a coherent manner. For example, one approach is to reanalyse the schemes to homogenise their differences, which are made subsidiary to a main scheme.

\subsection{Reanalysis}

In contrast to the direct analysis, we reanalyse the four schemes as subsidiary to or dependent on a single main scheme, the Argument from Credible Source (CS), which we articulate below; each of the four dependent schemes is a way of establishing a premise of the main scheme. In other words, the CS along with particular additional arguments for premises form a tree-like structure, where one (subsidiary) argument justifies the premise of another argument. This can be seen as an overall argument 
with subarguments, where the subarguments are glued together by unification of variables along with sortal restrictions on terms and predicates. The idea is that we can have a cascade of rules, where more specialised rules justify predicates that can unify with predicates in the body of some more abstract rule. For instance, schemes for eyewitness testimony or testimony from videotape may be used to justify predicates in a more generalised scheme for witness testimony; in turn, witness testimony can be used to justify predicates in yet a more abstract scheme for credible source. Our analysis extracts conceptual redundancy from the current analysis of schemes and formally homogenises them.

An analysis along these lines is compatible with a Logic Programming paradigm: a predicate $\mathcal{P}_{i}$ in the body of a rule $\mathcal{R}_{i}$ unifies with the predicate $\mathcal{P}_{j}$ that is the head of some other rule $\mathcal{R}_{j}$; the predicate $\mathcal{P}_{j}$ has to be justified (true) with respect to the rule $\mathcal{R}_{j}$, and by the same token we can say that $\mathcal{R}_{j}$ justifies $\mathcal{P}_{i}$. There may be alternative ways to justify $\mathcal{P}_{i}$, given different rules $\mathcal{R}_{k}, \ldots, \mathcal{R}_{l}$ with a predicate in the head that can unify with $\mathcal{P}_{i}$. As well, there may be rules that justify other predicates that are in the body of the rule $\mathcal{R}_{i}$. Similar points can be made for justifications of predicates in the body of $\mathcal{R}_{j}$. In the following, we show such a cascade.

We first formalise a main scheme Credible Source (CS), followed by formalisations of the subsidiary schemes, and show how we can relate the schemes set theoretically to yield the tree-like structure. We give the CS in two forms - Levels 6 and 2.

\section{Credible Source (CS).}

- John is a credible source about the domain of ornithology.

- John says that female blackbirds are brown.

- That female blackbirds are brown is a statement in the domain of ornithology.

- Therefore, presumptively, female blackbirds are brown.

Functional roles and typed propositional functions.

- credibleSourceAbout-Premise $F\left(X_{\text {argument }}\right)=\operatorname{credibleSourceAbout~}\left(Y_{\text {person }}, V_{\text {domain }}\right)$

- assertion-Premise $F\left(X_{\text {argument }}\right)=\operatorname{asserts}\left(Y_{\text {person }}, Z_{\text {literal }}\right)$

- statementInDomain-Premise $F\left(X_{\text {argument }}\right)=\operatorname{statementInDomain}\left(Z_{\text {literal }}, V_{\text {domain }}\right)$

- credibleSource-Concludes $F\left(X_{\text {argument }}\right)=Z_{\text {literal }}$

From our four previous schemes (PK, WT, EO, P), we take these as different ways of arguing for the CS premise credibleSourceAbout; that is, each of these schemes has as conclusion the propositional content associated with the credibleSourceAbout premise of the CS.

EO'

- expertAbout-Premise $F\left(X_{\text {argument }}\right)=\operatorname{expertAbout~}\left(Y_{\text {person }}, V_{\text {domain }}\right)$

- expertOpinion'-Concludes $F\left(X_{\text {argument }}\right)=$ credibleSourceAbout $\left(Y_{\text {person }}, V_{\text {domain }}\right)$ PK'

- positionToKnow-Premise $F\left(X_{\text {argument }}\right)=$ positionToKnow $\left(Y_{\text {person }}, V_{\text {domain }}\right)$

- positonToKnow'-Concludes $F\left(X_{\text {argument }}\right)=$ credibleSourceAbout $\left(Y_{\text {person }}, V_{\text {domain }}\right)$ $\mathbf{W T}^{\prime}$

- witnessConcerning-Premise $F\left(X_{\text {argument }}\right)=$ witnessConcerning $\left(Y_{\text {person }}, V_{\text {domain }}\right)$

- witnessTestimony'-Concludes $F\left(X_{\text {argument }}\right)=\operatorname{credibleSourceAbout}\left(Y_{\text {person }}, V_{\text {domain }}\right)$ $\mathbf{P}^{\prime}$

- perceivesConcerning-Premise $F\left(X_{\text {argument }}\right)=\operatorname{perceivesConcerning}\left(Y_{\text {person }}, V_{\text {domain }}\right)$

- perceives'-Concludes $F\left(X_{\text {argument }}\right)=\operatorname{credibleSourceAbout}\left(Y_{\text {person }}, V_{\text {domain }}\right)$ 
Clearly, $\mathrm{EO}^{\prime}, \mathrm{PK}^{\prime}, \mathrm{WT}^{\prime}$, and $\mathrm{P}^{\prime}$ all have the same propositional function as a conclusion, so they are all seem to be about making the same point, though they differ in how the premises justify the conclusion. More importantly, we can equate the conclusion of one AS (given a value for $\mathcal{Y}$ ) with the premise of another since the following is true:

$$
\begin{aligned}
\text { credibleSourceAbout-Premise } F\left(X_{\text {argument }}\right) & =\mathcal{Y} \text {-Concludes } F\left(X_{\text {argument }}\right) \\
& =\operatorname{credibleSourceAbout}\left(Y_{\text {person }}, V_{\text {domain }}\right)
\end{aligned}
$$

And in general, the premises and conclusions of ASs may be related as follows:

Definition 8 (Premise-conclusion tie).

$$
\mathcal{X} \text {-Premise } F\left(X_{\text {argument }}\right)=\mathcal{Y} \text {-Concludes } F\left(Y_{\text {argument }}\right)=V_{\text {literal }},
$$

where $\mathcal{X} \neq \mathcal{Y}$, and $X_{\text {argument }} \neq Y_{\text {argument }}$.

Consider a worked example. Suppose that we are making use of the $\mathrm{PK}^{\prime}, \mathrm{CS}$, and (for clarity) the equivalence of the premise and conclusion. Prior to instantiation, we have:

- $\mathbf{P K}^{\prime}$

- positionToKnow-PremiseF $\left(X_{\text {argument }}\right)=$ positionToKnow $\left(Y_{\text {person }}, V_{\text {domain }}\right)$

- positionToKnow'-Concludes $F\left(X_{\text {argument }}\right)=\operatorname{credibleSourceAbout}\left(Y_{\text {person }}, V_{\text {domain }}\right)$

\section{- CS}

- credibleSourceAbout-Premise $F\left(X_{\text {argument }}\right)=\operatorname{credibleSourceAbout}\left(Y_{\text {person }}, V_{\text {domain }}\right)$

- assertion-Premise $F\left(X_{\text {argument }}\right)=\operatorname{asserts}\left(Y_{\text {person }}, Z_{\text {literal }}\right)$

- statementInDomain-Premise $F\left(X_{\text {argument }}\right)=\operatorname{statementInDomain}\left(Z_{\text {literal }}, V_{\text {domain }}\right)$

- credibleSource-Concludes $F\left(X_{\text {argument }}\right)=Z_{\text {literal }}$

\section{- Premise-conclusion equivalence}

- credibleSourceAbout-Premise $F\left(X_{\text {argument }}\right)=$ positionToKnow'-Concludes $F\left(Y_{\text {argument }}\right)=$ credibleSourceAbout $\left(Y_{\text {person }}, V_{\text {domain }}\right)$

When we instantiate the schemes and unify the variables, the denotations of the schemes are tied together. Suppose that for a person john', a domain, ornithology', and a literal female_blackbirds_are_ brown', we have the following instantiation:

\section{- $\mathbf{P K}^{\prime}$}

- positionToKnow-PremiseF $\left(a_{i}\right)=$ positionToKnow $\left(\right.$ john $^{\prime}$, ornithology' $\left.^{\prime}\right)$

- positionToKnow'-Concludes $F\left(a_{i}\right)=$ credibleSourceAbout $\left(j o h n^{\prime}\right.$, ornithology' $\left.^{\prime}\right)$

\section{- CS}

- credibleSourceAbout-Premise $F\left(a_{i}\right)=$ credibleSourceAbout $\left(\right.$ john $^{\prime}$, ornithology')

- assertion-Premise $F\left(a_{i}\right)=$ asserts(john',female_blackbirds_are_brown')

- statementInDomain-PremiseF $\left(a_{i}\right)=$ statementInDomain(female_blackbirds_are_brown', ornithology')

- credibleSource-Concludes $F\left(a_{i}\right)=$ female_blackbirds_are_brown' 


\section{- Premise-conclusion equivalence}

- credibleSourceAbout-Premise $F\left(a_{i}\right)=$ positionToKnow ${ }^{\prime}$-Concludes $F\left(a_{i}\right)=$ credibleSourceAbout (john', ornithology')

From this, we see that with a contribution from $\mathrm{PK}^{\prime}$, the conclusion from CS is female_blackbirds_are_ brown'.

The other ASs - EO', $\mathrm{WT}^{\prime}, \mathrm{P}^{\prime}$ - could similarly be used. They are mutually compatible where we can unify the variables, giving different justifications for the same conclusions; where we have different unifications, then the resultant ASs may be understood as incompatible (or not, depending on other aspects of the given model). Each of these subsidiary ASs has a conclusion that matches a premise of the main scheme CS. We could have additional schemes to justify other premises of the CS, and so on, giving a rich tree made up of a main scheme with several subsidiary layers.

There is a range of lexical semantic issues to address. While our direct analysis may have introduced too fine-grained an analysis, the reconstruction here may have homogenised important distinctions in meaning, creating awkward expressions. For instance, is it correct to witness a domain or perceive a domain? These are different from the more familiar phrases of being an expert about a domain or being in a position to know about a domain. This suggests some further intermediate structure is required, where the CS has more abstract predicates that are argued for in more fine-grained ASs.

In our instantiation, we have but one argument individual $a_{i}$ which appears in two distinct ASs; this arises because of the unification of the argument variable. In [25], arguments have subarguments given the recursive syntactic definition of arguments. We could reproduce this semantically. Suppose that in the model, argument individuals are totally ordered by the subargument relation and that we have the following constraint:

Definition 9 (Subargument).

$$
\begin{aligned}
& {\left[\mathcal{X} \text {-Premise } F\left(X_{\text {argument }}\right)=\mathcal{Y} \text {-Concludes } F\left(Y_{\text {argument }}\right)=V_{\text {literal }}\right]} \\
& \quad \rightarrow \operatorname{subargument}\left(X_{\text {argument }}, Y_{\text {argument }}\right), \quad \text { where } \mathcal{X} \neq \mathcal{Y}, \text { and } X_{\text {argument }} \neq Y_{\text {argument }} .
\end{aligned}
$$

One $A S_{i}$ is a subargument of another $A S_{j}$, where one of the premises of $A S_{i}$ is the same literal as the conclusion of $A S_{j}$; that is, a subargument arises where the instantiation of the conclusion of one AS unifies with the premise of the instantiation of a different AS. This is not as in [25], where subarguments are defined recursively such that each branch of an argument tree is a subargument and so is each branch of each branch and so on. To capture a similar idea in our analysis, we assume the Transitive Closure of the subargument relation. The problem for us is the precise meaning, use, and importance of the recursive definition in ASPIC+, particularly where argument attacks are concerned. For our purposes, we leave investigation of these matters for future research.

\section{Discussion and future work}

In this paper, we have presented a functional language for a computational analysis of ASs that is compatible with argumentation frameworks. We have outlined an extensible methodology, worked through an example, and shown how ASs in our analysis can be systematically related to one another. In this section, we discuss some related work, topics that were not addressed in the presentation, and future work. 
There is an abundance of research in ASs. [36] provide a catalogue of ASs at a largely descriptive and unsystematic level. A range of considerations about ASs are developed in [26], but these do not give rise to a formal analysis. Some research remains at Level 5, where strings are annotated with respect to labels [32]. There are computational proposals which do not differentiate premises [25,30], while others do differentiate premises, but not with respect to the content of the associated propositions, e.g. [15]. ASs have been analysed for legal argumentation. In [34], an approach to the analysis of schemes is outlined and exemplified for legal reasoning. There are formalised ASs with propositional functions for legal case-based reasoning [7,38,39], though these are highly specialised. Moreover, the approach to abstracting arguments is underspecified and not tied to a theory of argumentation, e.g. ASPIC + . None of these proposals for legal reasoning propose a more general language for ASs. A different line of work provides an interchange format and associated ontology [29], which allows for differentiated premises; however, this work does not tie the premise subsorts to the propositional content, much less to the natural language statements of the argument; nor does it take into account formal argumentation systems. In [10], the formal problems with the AIF are addressed by interpreting it in terms of ASPIC + . Our approach is distinct from ASPIC+ [25] in several ways, where our proposal: has heterogeneous premises; ties the role of a premise in an AS to the propositional content of the premise; differentiates generic reasoning patterns from ASs; uses predicate logical structure; and relates the natural language expression of an AS to a formal, argumentation-theoretic representation. Closest in spirit to our proposal is [4,5], where a natural language expression of practical reasoning is analysed in terms of predicates and terms with respect to denotations in a semantic model. In that approach, ASs are construed as arguments, though the specific translation is underspecified and a language for ASs is not provided. Also related to our proposal is the Carneades system; the features of the current implementation are given in [14]. The similarities are not accidental: like our work, Carneades originates in the ESTRELLA and IMPACT Projects, ${ }^{12}$ although Carneades remains essentially a separate development of an implementation designed to support different workpackages in those projects.

In Sections 4.2 and 4.3, we pointed to issues about the fine-grainedness of premise functions. Finegrainedness arises as there are many ways of expressing or modifying a predicate: one may know indirectly or know in one's heart; one may assert quietly or state or assert in court. Similarly, the terms of the predicates may vary with respect to properties of the person or alternative forms of the proposition. In general, this is related to similar discussions about propositions and propositional attitudes, e.g. belief, and may warrant a similar treatment [12]. One way to treat some of these issues is to homogenise terminology under synonyms, e.g. asserts in the data is substitutable by said or wrote, so we can presume these map to the same premise function. The knowledge base may be further enriched for such lexical semantic information. Alternatively, one may simply use a controlled language [37] to constrain the expressivity of the language in a given domain. However, this is a general topic that requires significant further research.

In future work, we look forward to a range of topics. Of a particular interest is the relationship of ASs to semantic models, critiques, and dialogue. Current examples of semantic models are the Knowledge bases of [25] and the transition systems of [4,5]. We have not discussed the dialogical aspects of argumentation, nor critical questions, which are questions used to critique the presumptive truth of the instantiated AS. These topics are closely related, crucial aspects to a full analysis of ASs and argumentation. Briefly, ASs may be critiqued, using critical questions, in the course of a dialogue, where there are

\footnotetext{
${ }^{12}$ ESTRELLA Project (IST-2004-027655): http://www.estrellaproject.org/. IMPACT Project (FP7-ICT-2009-4): http://www. policy-impact.eu/.
} 
alternative instantiations of propositions of the AS relative to the semantic model; the questions encode these alternatives. Models are also used to generate instantiated ASs, some of which may be in attack relations (cf. on these topics [4,5]). In analysing a range of ASs, of special interest is the differentiation of semantic models with respect to the requirements of the scheme; in other words, the different fragments of the language are given appropriate semantic models. In [25], three different knowledge bases are provided, but it differentiates only between facts, strict rules and defensible rules, rather than supporting the many sorts required by our analysis. We want to differentiate sorts of rules and justify them accordingly, so that, e.g. rules about causation, definition, observational generalisation, values, assertions, are each given a distinct sort of justification (and related attacks), and the meanings of the justifications (attacks) are grounded in an associated semantic model. With such analyses, we could look to implement ASs in a database for web-based applications [40] and Functional or Logic Programs that instantiate the ASs, as well as to generate arguments, calculate attacks, and determine extensions.

One final research direction is to investigate the relationships between our formalisation of argumentation schemes with ongoing work in argumentation mining [17], where arguments in unstructured natural language corpora are extracted and mapped to abstract arguments for reasoning in Dungian AFs. Current techniques apply machine learning to identify topics, classify statements, and relate contrastive statements. Yet, given the complexity of natural language, current mining approaches do not appear to account for synonymy, contradiction, or entailment, as these require rich domain and linguistic information along with fine-grained syntactic and semantic analysis into a formal language. While our approach to argumentation schemes also cannot yet be used for argumentation mining, it does provide a theoretical "target" for such mined arguments, e.g. the normalised expressions and their relationships. In this sense, our work is the theoretical framework for mining argumentation schemes.

\section{Acknowledgements}

This work is based on research done at University of Liverpool on the FP7-ICT-2009-4 Programme, IMPACT Project, Grant Agreement Number 247228. The Principal Investigator at Liverpool was Katie Atkinson. I particularly thank Professor Trevor Bench-Capon for many discussions and contributions on this topic. The paper is a substantive revision of a paper presented at the Ninth International Workshop on Argumentation in Multi-Agent Systems (ArgMAS 2012).

\section{References}

[1] L. Amgoud and C. Cayrol, On the acceptability of arguments in preference-based argumentation, in: Proceedings of the 14th Annual Conference on Uncertainty in Artificial Intelligence (UAI-98), Morgan Kaufmann, San Francisco, CA, 1998, pp. $1-7$.

[2] L. Amgoud, C. Devred and M. Lagasquie-Schiex, A constrained argumentation system for practical reasoning, in: Argumentation in Multi-Agent Systems, Fifth International Workshop, ArgMAS 2008, Revised Selected and Invited Papers, Vol. 12, Estoril, Portugal, 2008, pp. 37-56.

[3] N. Asher, Reference to Abstract Objects in Discourse, Kluwer Academic Publishers, 1993.

[4] K. Atkinson, T. Bench-Capon, D. Cartwright and A. Wyner, Semantic models for policy deliberation, in: Proceedings of the Thirteenth International Conference on Artificial Intelligence and Law (ICAIL 2011), Pittsburgh, PA, USA, 2011, pp. 81-90.

[5] K. Atkinson and T.J.M. Bench-Capon, Practical reasoning as presumptive argumentation using action based alternating transition systems, Artificial Intelligence 171(10-15) (2007), 855-874. doi:10.1016/j.artint.2007.04.009.

[6] T. Bench-Capon and K. Atkinson, Argumentation schemes: From informal logic to computational models, in: Dialogue and Argumentation: An Examination of Douglas Waltonś Theories of Reasoning and Argument, C. Reed and C. Tindale, eds, Academic Press, London, 2010, pp. 103-114. 
[7] T. Bench-Capon and H. Prakken, Using argument schemes for hypothetical reasoning in law, Artificial Intelligence and Law 18(2) (2010), 153-174. doi:10.1007/s10506-010-9094-8.

[8] T.J.M. Bench-Capon, Persuasion in practical argument using value-based argumentation frameworks, Journal of Logic and Computation 13(3) (2003), 429-448. doi:10.1093/logcom/13.3.429.

[9] P. Besnard and A. Hunter, Elements of Argumentation, MIT Press, 2008.

[10] F. Bex, H. Prakken and C. Reed, A formal analysis of the AIF in terms of the ASPIC framework, in: Proceedings of the 2010 Conference on Computational Models of Argument: Proceedings of COMMA 2010, IOS Press, Amsterdam, The Netherlands, 2010, pp. 99-110.

[11] G. Chierchia and S. McConnell-Ginet, Meaning and Grammar: An Introduction to Semantics, MIT Press, 2000.

[12] M.J. Cresswell, Structured Meanings: The Semantics of Propositional Attitudes, Bradford Books. MIT Press, 1985.

[13] L.T.F. Gamut, Logic, Language, and Meaning: Intensional Logic and Logical Grammar, University of Chicago Press, 1991.

[14] T. Gordon, The Carneades web service, in: Proceedings of the Fourth International Conference on Computational Models of Argument (COMMA 2012), B. Verheij, S. Szeider and S. Woltran, eds, IOS Press, Amsterdam, 2012, pp. 517-518.

[15] T. Gordon, H. Prakken and D. Walton, The Carneades model of argument and burden of proof, Artificial Intelligence 171 (2007), 875-896. doi:10.1016/j.artint.2007.04.010.

[16] N. Lavrač, I. Bratko, I. Mozetič, B. Čerček, A. Grad and M. Horvat, KARDIO-E: An expert system for electrocardiographic diagnosis of cardiac arrhythmias, Expert Systems 2(1) (1985), 46-55. doi:10.1111/j.1468-0394.1985.tb00449.x.

[17] M. Lippi and P. Torroni, Argumentation mining: State of the art and emerging trends, ACM Transactions on Internet Technology 16(2) (2016), 10:1-10:25. doi:10.1145/2850417.

[18] R.P. Loui and J. Norman, Rationales and argument moves, Artificial Intelligence and Law 3(3) (1995), 159-189. doi:10.1007/BF00872529.

[19] J. McCarthy, Circumscription - A form of non-monotonic reasoning, Artificial Intelligence 13 (1980), 27-39. doi:10.1016/0004-3702(80)90011-9.

[20] J. McCarthy, Applications of circumscription to formalizing common sense knowledge, Artificial Intelligence Journal 28 (1986), 89-116. doi:10.1016/0004-3702(86)90032-9.

[21] R. Montague, The proper treatment of quantification in ordinary English, in: Formal Philosophy: Selected Papers of Richard Montague, R. Thomason, ed., Yale University Press, 1974, pp. 247-270.

[22] C. Perelman and L. Olbrechts-Tyteca, The New Rhetoric: A Treatise on Argumentation, University of Notre Dame, 1958. Translated into English by John Wilkinson and Purcell Weaver in 1969.

[23] P.M. Dung, On the acceptability of arguments and its fundamental role in nonmonotonic reasoning, logic programming and n-person games, Artificial Intelligence 77(2) (1995), 321-358. doi:10.1016/0004-3702(94)00041-X.

[24] Phan, M. Dung, R. Kowalski and F. Toni, Assumption-based argumentation, in: Argumentation in Artificial Intelligence, Springer, 2009, pp. 199-218.

[25] H. Prakken, An abstract framework for argumentation with structured arguments, Argument and Computation 1(2) (2010), 93-124. doi:10.1080/19462160903564592.

[26] H. Prakken, On the nature of argument schemes, in: Dialectics, Dialogue and Argumentation. An Examination of Douglas Walton's Theories of Reasoning and Argument, C. Reed and C. Tindale, eds, College Publications, London, 2010, pp. 167185.

[27] I. Rahwan and L. Amgoud, An argumentation based approach for practical reasoning, in: AAMAS, 2006, pp. $347-354$. doi:10.1145/1160633.1160696.

[28] I. Rahwan, B. Banihashemi, C. Reed, D. Walton and S. Abdallah, Representing and classifying arguments on the semantic web, Knowledge Engineering Review 26(4) (2011), 487-511. doi:10.1017/S0269888911000191.

[29] I. Rahwan and C. Reed, The argument interchange format, in: Argumentation in Artificial Intelligence, G. Simari and I. Rahwan, eds, Springer, US, 2009, pp. 383-402. doi:10.1007/978-0-387-98197-0_19.

[30] C. Reed and D. Walton, Towards a formal and implemented model of argumentation schemes in agent communication, Autonomous Agents and Multi-Agent Systems 11 (2005), 173-188. doi:10.1007/s10458-005-1729-x.

[31] R. Reiter, A theory of diagnosis from first principles, Artificial Intelligence 32(1) (1987), 57-95. doi:10.1016/00043702(87)90062-2.

[32] G. Rowe and C. Reed, Argument diagramming: The Araucaria Project, in: Knowledge Cartography: Software Tools and Mapping Techniques, A. Okada, S.B. Shum and T. Sherborne, eds, Springer, 2008, pp. 163-181.

[33] Y. Tang and S. Parsons, Argumentation-based multi-agent dialogues for deliberation, in: Argumentation in Multi-Agent Systems, Second International Workshop, ArgMAS 2005, S. Parsons, N. Maudet, P. Moraitis and I. Rahwan, eds, Utrecht, The Netherlands, July 26, 2005, Lecture Notes in Computer Science, Vol. 4049, Springer, 2005, pp. 229-244. Revised Selected and Invited Papers.

[34] B. Verheij, Dialectical argumentation with argumentation schemes: An approach to legal logic, Artificial Intelligence and Law 11 (2003), 167-195. doi:10.1023/B:ARTI.0000046008.49443.36.

[35] D. Walton, Argumentation Schemes for Presumptive Reasoning, Erlbaum, Mahwah, N.J., 1996. 
[36] D. Walton, C. Reed and F. Macagno, Argumentation Schemes, Cambridge University Press, 2008.

[37] A. Wyner, K. Angelov, G. Barzdins, D. Damljanovic, B. Davis, N. Fuchs, S. Hoefler, K. Jones, K. Kaljurand, T. Kuhn, M. Luts, J. Pool, M. Rosner, R. Schwitter and J. Sowa, On controlled natural languages: Properties and prospects, in: Proceedings of the 2009 Conference on Controlled Natural Language, CNL'09, Springer-Verlag, Berlin, Heidelberg, 2010, pp. 281-289.

[38] A. Wyner and T. Bench-Capon, Argument schemes for legal case-based reasoning, in: Legal Knowledge and Information Systems. JURIX 2007, A.R. Lodder and L. Mommers, eds, IOS Press, Amsterdam, 2007, pp. 139-149.

[39] A. Wyner, T. Bench-Capon and K. Atkinson, Towards formalising argumentation about legal cases, in: Proceedings of the Thirteenth International Conference on Artificial Intelligence and Law (ICAIL 2011), Pittsburgh, PA, USA, 2011, pp. $1-10$.

[40] A.Z. Wyner, K. Atkinson and T. Bench-Capon, Towards a structured online consultation tool, in: Electronic Participation Third International Conference (ePart 2011), Lecture Notes in Computer Science (LNCS), Vol. 6847, Springer, Berlin, Germany, 2011, pp. 286-297. 